\title{
WA105: A Large LAr Double Phase TPC Prototype
}

\author{
Thorsten Lux ${ }^{1, a}$ on behalf of the WA105 collaboration \\ ${ }^{1}$ Institut de Física d'Altes Energies (IFAE) - The Barcelona Institute of Science and Technology, Campus UAB, Bellaterra \\ (Barcelona), Spain
}

\begin{abstract}
For the next generation of long baseline neutrino oscillation experiments detectors with several tens of ktons of detection medium will be needed. Liquid argon (LAr) is ideal for this application since it allows the construction of fully sensitive time projection chambers (TPCs). A mandatory step between the current setups and the final detector for the long baseline experiment is the construction of a large prototype to prove the technical feasibility of the scaling. WA105 with a size of $6 \times 6 \times 6 \mathrm{~m}^{3}$ is this intermediate step for the detector concept of a LAr double phase TPC and will be presented in these proceedings.
\end{abstract}

\section{Introduction}

The next generation of long baseline neutrino oscillation experiment, the Deep Underground Neutrino Experiment (DUNE), foresees as detector technology for the far detector 4 liquid argon (LAr) time projection chambers (TPC) of about 10 kton sensitive mass each. LAr has the advantage to provide the required large detector masses at reasonable costs and to allow the construction of a fully active tracking calorimeter. The basic principle of a LAr TPC is the following: A charged particle traversing the LAr ionizes the argon atoms creating a large amount of electron-ion pairs on its way through the gas. For example minimal ionizing particles like cosmic muons create in 1 $\mathrm{cm}$ about 50.000 electron-ion pairs. By applying an external electric field, the drift field, the electrons and ions are separated and drift to the the anode and cathode respectively. The field strength of the drift field is normally chosen to be between 0.5 and $1 \mathrm{kV} / \mathrm{cm}$. While the ions are simply neutralized at the cathode, the electrons measured at the anode are used to reconstruct the event. Two different detector concepts exist for the anode side. In the single phase detector a set of wires is immersed in the liquid on which the electrons create a signal, either due to induction or direct absorption. In the double phase detector the electrons are extracted from the liquid to a gaseous volume within a strong electric field of 2 to $3 \mathrm{kV} / \mathrm{cm}$ and undergo afterwards a charge amplification before the signal is read out at the segmented anode. While the first detector concept is simpler, the double phase concept has several advantages: LAr is critically sensitive to oxygen impurities which causes the loss of electrons while drifting to the anode as illustrated in Fig. 1 (left). Due to the amplification in the gas phase these losses can be partly compensated allowing lower energy detection thresholds and simpler electronics. The signals recorded at the segmented anode

\footnotetext{
a e-mail: Thorsten.Lux@ifae.es
}

allow to reconstruct the trajectory of the charged particle in 3D. However, the signal measured at the anode does not provide information about the absolute $z$ coordinate, the position between anode and cathode, of the event. This information can be obtained from the primary scintillation light which is emitted isotropically during the ionization process. Measuring the time difference between this light pulse and the arrival of the electrons at the anode provides the $z$ coordinate assuming the knowledge of the electron drift velocity. The concept of a LAr double phase TPC is shown in Fig. 1 (right).

The aim of the WA105 project [1] is the construction of a large double phase LAr TPC prototype to test all technological aspects necessary for the scaling to kton detectors. In addition the detector will be characterized using a well defined testbeam. In the following the WA105 detector, the testbeam characteristics and the time schedule will be presented in more detail.

\section{The WA105 Detector}

The design of the WA105 detector is shown in Fig. 2. The cryostat has walls $1 \mathrm{~m}$ thick which limit the heat transfer to maximal $5 \mathrm{~W} / \mathrm{m}^{2}$. Its inner volume has dimensions of $8.3 \times 8.3 \times 8.1 \mathrm{~m}^{3}(\mathrm{WxLxH})$ containing about 700 ton of LAr. The sensitive volume, defined by the field cage, anode and cathode, has dimensions of $6 \times 6 \times 6 \mathrm{~m}^{3}$ and contains about 300 ton of active mass. The large depths of the detector lead to voltages of up to $600 \mathrm{kV}$ at the cathode which consists of wires to achieve a high transparency for the photons of the primary scintillation light. A set of 36 PMTs with a diameter of 8" (Hamamatsu R5912-02mod) is installed about $1 \mathrm{~m}$ below the cathode. Since the PMTs are not directly sensitive to the $128 \mathrm{~nm}$ of the primary scintillation light, acrylic plates coated with tetraphenyl butadine (TPB) will be installed directly in front of the PMTs. TPB shifts the light from $128 \mathrm{~nm}$ to about $430 \mathrm{~nm}$, 

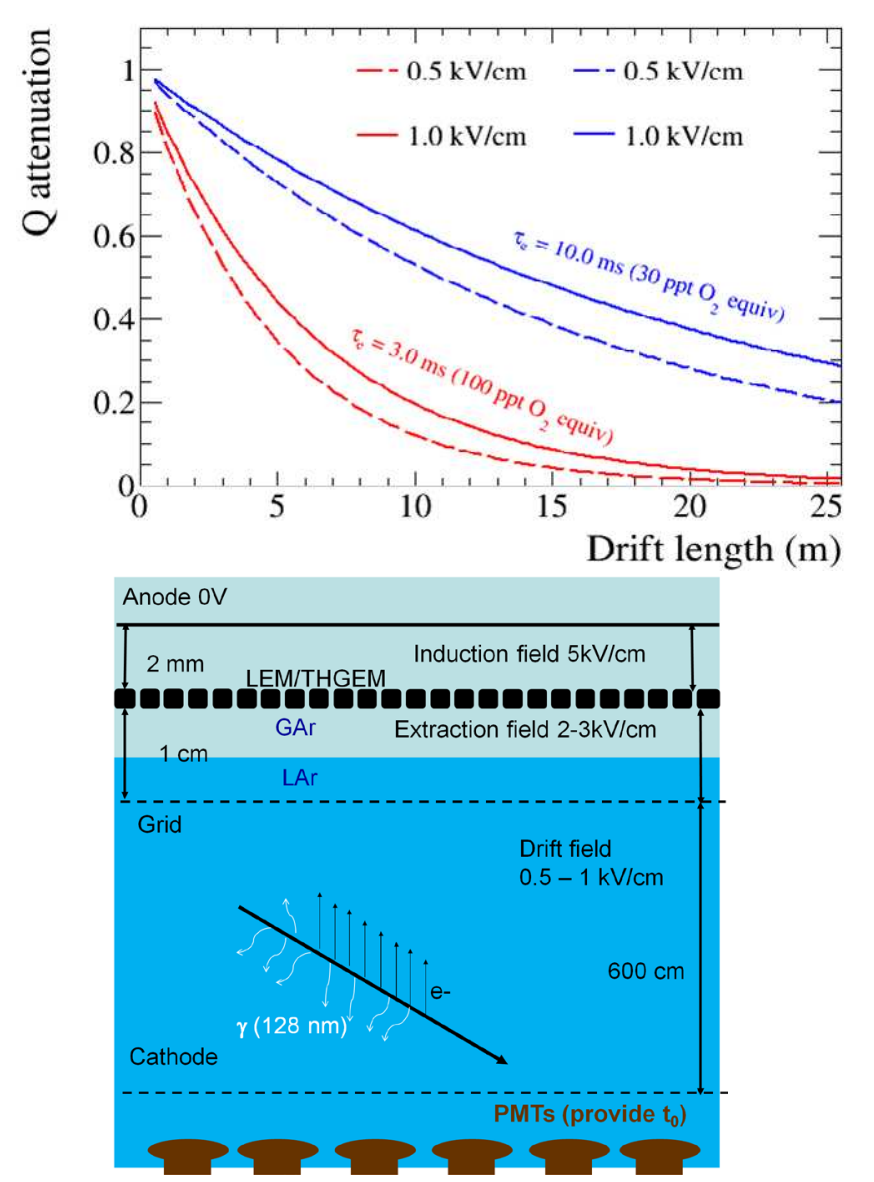

Figure 1. The effect of oxygen contamination on the charge loss (left). The principle of a double phase LAr (right).

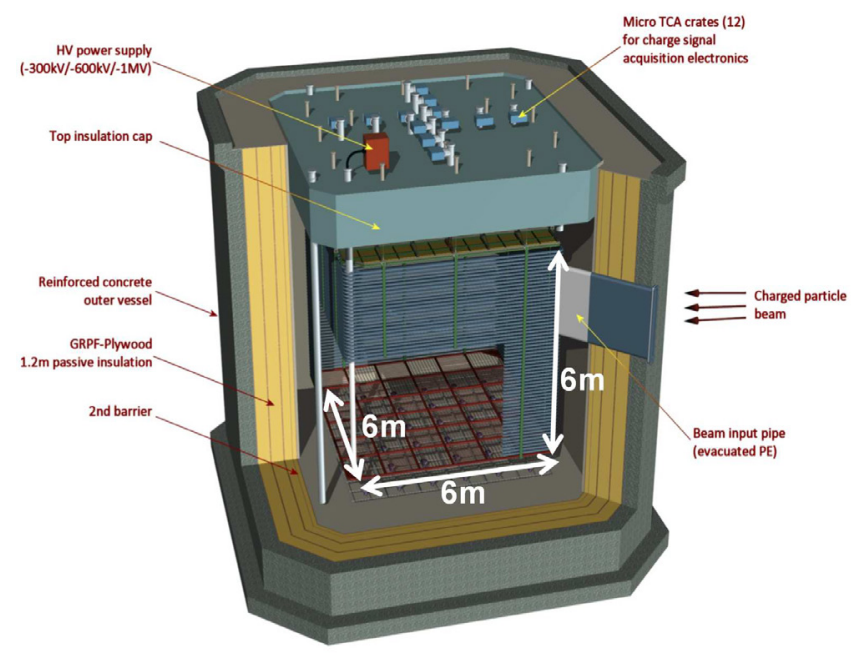

Figure 2. Schematic view of the WA105 detector. The sensitive volume has dimensions of $6 \times 6 \times 6 \mathrm{~m}^{3}$ and contains about 300 ton of LAr. a wavelength at which the PMTs highly sensitive. On the anode side the electrons are extracted from the liquid to the gaseous phase applying an electric field of $3 \mathrm{kV} / \mathrm{cm}$ at the phase transition zone. For this a steel mesh with wire diameter of $100 \mu \mathrm{m}$ and wire pitch of $3.125 \mathrm{~mm}$ is immersed $5 \mathrm{~mm}$ into the liquid phase. In the gaseous phase, $5 \mathrm{~mm}$ above the liquid surface, Large Electron Multipliers (LEMs) are installed. A LEM is a printed circuit board (PCB), $1 \mathrm{~mm}$ thick, coated on both sides with copper and highly perforated by drilled holes. The diameter of the holes in the PCB is $500 \mu \mathrm{m}$ and the holes are distributed in a hexagonal pattern with $800 \mu \mathrm{m}$ pitch. The holes in the copper have a $40 \mu \mathrm{m}$ larger radius to improve HV stability. By applying a voltage between the two copper sides, the electrons extracted from the liquid are guided into the holes where the electric field is so high that charge amplification occurs. The performance of LEMs was intensively studied in pure Ar at cryogenic temperatures [2]. It was found that at a voltage difference of about $3500 \mathrm{~V} / \mathrm{cm}$, an effective gain of 20 can be achieved. The amplified electrons are drifting afterwards towards the anode. The gap between LEM and anode is $2 \mathrm{~mm}$. The anode is segmented in strips in $x$ and and $y$, providing two independent views. The pitch of the strips is $3.125 \mathrm{~mm}$ and each strip has a length of $3 \mathrm{~m}$. Their geometry is optimized to achieve equal charge share between the two views and to minimize parasitic capacitance between adjacent strips. In total there will be 7680 strips, 3840 for each view. The signals are extracted on the top of the cryostat by a system of feedthrough chimneys which reach into the gaseous phase to minimize the cable length between anode and analog preamplifier which are installed at the bottom of the chimneys, outside of the cryostat inner volume. This arrangement is possible thanks to the pre-amplification in the gaseous argon and is a clear advantage of the double phase concept since it allows the maintenance of the electronics without the need to open the cryostat. The analog preamplifiers are based on CMOS technology and are optimized in respect of low power consumption, $18 \mathrm{~mW}$ per channel, and low noise. For latter also the ambient temperature of $110 \mathrm{~K}$ is an advantage. The digital part of the electronics is installed on top of the chimneys in $\mu \mathrm{TCA}$ crates. The principle design is shown in Fig. 3. The signals are digitized with a sampling rate of $2.5 \mathrm{MHz}$ over a time period of $4 \mathrm{~ms}$ per event given my the maximal drift time of the electrons. Two trigger modes are implemented in the electronics: for the testbeam data taking an external trigger synchronized with the arrival of the particles is used, while data taking with cosmics is performed with a self-triggering mode based on the PMT signals.

\section{Location and Testbeam}

The WA105 detector will be installed in the extension of the EHN1 testbeam facility, currently under construction, at the CERN Prevessin site. This location allows to expose the detector to a beam consisting of muons, electrons and pions of both polarities. The energy of the beam is between 0.4 and $10 \mathrm{GeV} / \mathrm{c}$. The beam allows studying 
the detector performance as hadronic and electromagnetic calorimeter, the particle identification capability of the detector and especially the e $/ \pi^{ \pm}$separation. In addition it enables the possibility to develop and optimize reconstruction software and its validation.

\section{Conclusions and Outlook}

For the next generation of neutrino oscillation experiment detectors with about 10 kton active mass will be needed. The WA105 detector is a necessary intermediate step for these detectors to prove their technical feasibility and will provide also a deeper understanding of the detector performance due to its exposing to a well defined beam of particles at CERN. The WA105 collaboration consisting of about 120 physicists from more than 20 institutes and 10 countries is working towards the construction of a large

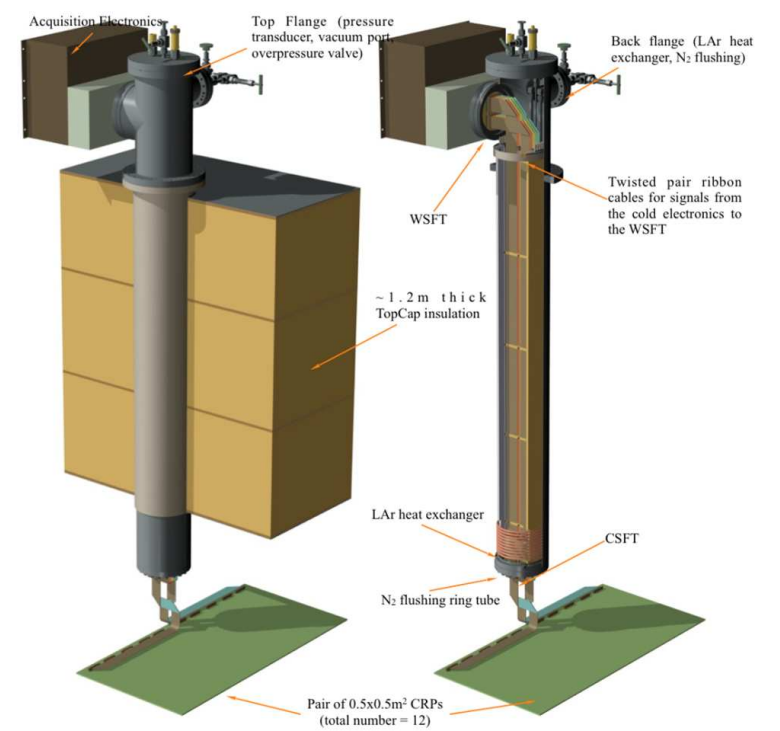

Figure 3. Conceptual drawing of the chimney approach. The analog preamplifier are installed at the bottom of the chimneys, outside of the inner cryostat volume, while the digitial electronics is mounted on top of the chimneys. double phase LAr TPC with an active mass of about 300 ton. The start of the data taking is foreseen for 2018 .

\section{Acknowledgment}

The author acknowledge the support received from the Ministerio de Ciencia e Innovacion under grants FPA2014-59855-P, TEC2012-39150-C02-02 and Centro de Excelencia Severo Ochoa SEV-2012-0234, some of which include ERDF funds from the European Union.

\section{References}

[1] L. Agostino et al., SPSC-TDR-004, arXiv: 1409.4405 (2014)

[2] C. Cantini et al, JINST 9 P03017, arXiv: 1312.648 (2014) 